\title{
ON THE ELEMENT OF DECOMPOSITION OF A DOUBLY PERIODIC FUNCTION OF THE SECOND KIND*
}

\author{
BY M. A. BASOCO
}

1. Introduction. Hermite $\dagger$ has shown that a meromorphic function which satisfies periodicity relations of the form

$$
F(z+2 \omega)=\mu F(z), \quad F\left(z+2 \omega^{\prime}\right)=\mu^{\prime} F(z),
$$

where $\omega^{\prime} / \omega=a+i b, b>0$, and $\mu, \mu^{\prime}$ are independent of $z$, may be expressed in terms of the function

$$
G(z)=\frac{\sigma(z+\lambda)}{\sigma(z) \sigma(\lambda)} e^{\rho z},
$$

and its derivatives, in which $\lambda, \rho$ are suitably determined constants and $\sigma(u)$ is the Weierstrass sigma function. The class of functions which satisfy conditions (1) has been called by Hermite doubly periodic of the second kind. We shall exclude from the present considerations Mittag-Leffler's singular case $\ddagger$ for this category of functions. He has shown that if $F(z)=f(z) e^{\rho z}$, where $f(z)$ is an elliptic function, then the suitable element of decomposition is $e^{\rho z} \zeta(z)$, where $\zeta(z)$ is the zeta function of Weierstrass.

In what follows we shall be concerned with the nature of the power series development $\S$ of (2) which we shall henceforth write in the form

$$
\Phi(u, v)=\frac{\sigma(u+v)}{\sigma(u) \sigma(v)} e^{\rho z},
$$

* Presented to the Society, June 23, 1933.

$\dagger$ Hermite, Sur quelques applications des fonctions elliptiques, Oeuvres, vol. 3, Chaps. 1, 2, pp. 266 et seq. See also the monograph of Appell, Sur la decomposition d'une fonction méromorphe en éléments simples, Mémorial des Sciences Mathématiques, Fascicule 36, 1929.

† Comptes Rendus, vol. 90 (1880), p. 178.

§ See Krause, Doppeltperiodischen Funktionen, vol. 1, pp. 299-308. Halphen, Fonctions Elliptiques, vol. 1, Chap. 7. 
where $u, v$ are to be regarded as independent complex variables. For suitable selections of $\rho$, various expressions are obtained which are of importance in the applications. Thus, if $\rho=-\zeta(v)$ or $x-\zeta(v)$, the corresponding $\Phi(u, v)$ is fundamental in certain physical problems, as will be evident from even a cursory examination of the second volume of Halphen's treatise. For $\rho=-\eta / \omega,(\eta=\zeta(\omega))$, the function (3) is essentially the same as $\vartheta_{1}^{\prime} \vartheta_{1}(x+y) /\left\{\vartheta_{1}(x) \vartheta_{1}(y)\right\}$, which is of importance in some of the applications of doubly periodic functions to number theory.

It will be sufficient to treat in detail the case $\rho=x-\zeta(v)$; the results for the other cases mentioned being obtained on setting $x=0, x=Z(v)$, respectively, where this last is the zeta function of Hermite. ${ }^{*}$ We find that, for this generic case, the expansion (3) in a power series in $u$ has coefficients which are polynomials in $x$ with elliptic functions of $v$ as coefficients. As might be expected, these polynomials belong to the class of Appell polynomials $\dagger$ and can be constructed by recurrence. They will be shown to satisfy a certain linear differential equation.

2. The Generator $\phi$. The generator for the polynomials in question is the function

$$
\phi=\frac{\sigma(u+v)}{\sigma(u) \sigma(v)} e^{-u \zeta(v)},
$$

which, as may be seen from the properties of the sigma function, has a power series expansion about the origin $u=0$, of the form

$$
\begin{aligned}
\phi= & \frac{1}{u}\left\{1+\sum_{n=1}^{\infty} \alpha_{n} \frac{u^{n}}{n !}\right\} \\
& \left(\alpha_{1}=0, \alpha_{2}=-\wp(v), \alpha_{3}=-\wp^{\prime}(v), \text { etc. }\right) .
\end{aligned}
$$

It follows from the theory of Appell polynomials that the function

$$
\psi=\phi e^{x u}=\frac{\sigma(u+v)}{\sigma(u) \sigma(v)} e^{(x-\zeta(v)) u}
$$

when expanded in a power series in $u$ will have as the coeffi-

\footnotetext{
* See Appell-Lacour, Fonctions Elliptiques, p. 27.

$\dagger$ Appell, Sur une classe de polynômes, Annales de l'École Normale Supérieure, 1880.
} 
cient of the general term a polynomial $A_{n}(x)$ which symbolically may be represented by $(x+\alpha)^{n}$, where $\alpha$ is the umbra of the sequence $\alpha_{1}, \alpha_{2}, \alpha_{3}, \cdots, \alpha_{n}, \cdots$. The binomial expansion for $A_{n}(x)$ is to be understood in the sense that the exponents of $\alpha$ are to be degraded into suffixes; thus, $\alpha^{k}$ is to be replaced by $\alpha_{k}$.

It can be readily shown* that the generator $\phi$ satisfies the Lamé differential equation

$$
\frac{\partial^{2} \phi}{\partial u^{2}}=(2 \wp(u)+\wp(v)) \phi
$$

where $\&(u)$ is the Weierstrass elliptic function.

3. The Function $\psi$. It follows from (6) and (7) that the function $\psi$ satisfies the partial differential equations

$$
\frac{\partial \psi}{\partial x}=u \psi
$$

$$
\frac{\partial^{2} \psi}{\partial u^{2}}-2 x \frac{\partial \psi}{\partial u}+x^{2} \psi=(2 \wp(u)+\wp(v)) \psi .
$$

We may assume that $\psi$ has a power series expansion of the form

$$
\psi=\frac{1}{u}+\sum_{n=0}^{\infty} A_{n+1}(x, v) \frac{u^{n}}{(n+1) !} .
$$

* Halphen, vol. 1, p. 235. In this connection it may not be out of place to point out a curious error which has crept into the literature. In a paper by E. Jahnke, Journal für Mathematik, vol. 112 (1893), pp. 265-286, there are given several results concerning differential equations satisfied by doubly periodic functions of the second and third kinds. The last section of the paper (Third part, p. 282) is devoted to a study of certain differential identities due to Caspary (Journal de Mathématiques, (4), vol. 6, pp. 367-404), which give rise precisely to our generator $\phi(u, v)$. Apparently, Jahnke transcribed Caspary's function (see Caspary's paper, p. 403) erroneously and makes the statement that the function $\phi(u, v) \exp (-v \zeta(u))$ satisfies our equation $(7)$ as well as certain others derivable from it. This, however, is not the case, as may be readily seen. Jahnke's results are correct for our generator $\phi(u, v)$ but are not true for the function

used by him.

$$
\frac{\sigma(u+v)}{\sigma(u) \sigma(v)} \exp (-u \zeta(v)-v \zeta(u))
$$


On substituting this expansion in (8), we obtain, as the result of equating the coefficients of like powers of $u$, the relation

$$
\frac{\partial A_{n}}{\partial x}=n A_{n-1}
$$

which verifies the fact that the $A_{n}(x, v)$ are Appell polynomials in $x$.

Next, if the development (10) is substituted in (9), and both sides of the equation are expressed as power series in $u$, we find on equating the coefficients of corresponding powers two recurrences on the $A_{n}$, one being derived from the odd powers, the other from the even. However, if we define $A_{0}=1$, these two recurrences may be combined into the following one:

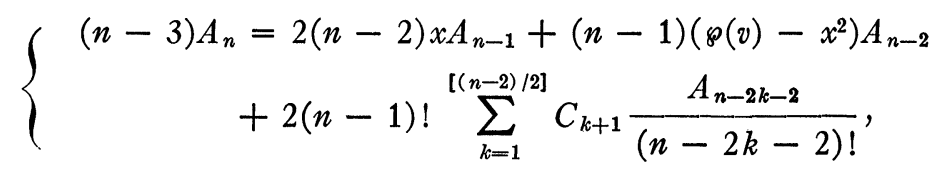

where

$$
C_{k}=\frac{3}{(2 k+1)(k-3)} \sum_{r=2}^{k-2} C_{r} C_{k-r}, \quad(k=4,5,6, \cdots),
$$

and, as usual, $[s / 2]$ is the greatest integer contained in $s / 2$. The $C_{k}$ are the coefficients in the expansion of the Weierstrass $\wp-$ function.

It is clear that the coefficients $\alpha_{k}$ of the polynomials $A_{n}$ are integral rational expressions in $\wp(v), \wp^{\prime}(v)$, and the fundamental invariants $g_{2}, g_{3}$ of $\wp(u)$, with rational coefficients. For example, we have

$$
\begin{aligned}
A_{0}=1 \text { (by definition), } A_{1}=x, \quad A_{2}=x^{2}-\wp(v), \\
A_{3}=x^{3}-3 \wp(v) x-\wp^{\prime}(v), \\
A_{4}=x^{4}-6 \wp(v) x^{2}-4 \wp^{\prime}(v) x-3 \wp^{2}(v)+(3 / 5) g_{2}, \\
A_{5}=x^{5}-10 \wp(v) x^{3}-10 \wp^{\prime}(v) x^{2}+5 x\left((3 / 5) g_{2}-3 \wp^{2}(v)\right) \\
\quad-2 \wp(v) \wp^{\prime}(v),
\end{aligned}
$$

If we let $x=0$, then recurrence (12) gives a simpler means of calculating the coefficients $\alpha_{k}$ in the generator $\phi$. Moreover, if $x=Z(v)=\zeta(v)-(\eta / \omega) v$, where $Z(v)$ is the zeta function of Hermite, we obtain the coefficients in the development of 


$$
\frac{\sigma(u+v)}{\sigma(u) \sigma(r)} e^{-(\eta / \omega) u v}
$$

which in terms of the Jacobi theta functions is*

$$
\frac{1}{2 \omega} \vartheta_{1}^{\prime} \frac{\vartheta_{1}(x+y)}{\vartheta_{1}(x) \vartheta_{1}(y)}, \quad x=\frac{u}{2 \omega}, \quad y=\frac{v}{2 \omega} \text {. }
$$

This function is of importance in the arithmetical applications.

The differential equation of which $A_{n}$ is a solution may be found readily by combining relations (11) and (12). Thus we obtain :

$$
\begin{aligned}
& 2 \sum_{k=1}^{[(n-2) / 2]} C_{k+1} \frac{\partial^{2 k+2} A_{n}}{\partial x^{2 k+2}}+\left(\wp(v)-x^{2}\right) \frac{\partial^{2} A_{n}}{\partial x^{2}}+2(n-2) \frac{\partial A_{n}}{\partial x} \\
& \quad-n(n-3) A_{n}=0,
\end{aligned}
$$

which is of order $n$ or $n-1$ according as $n$ is even or odd.

4. The Polynomials $\Gamma_{n}$. A system of Appell polynomials $\Gamma_{n}$ analogous to the ones just discussed may be obtained from the generator

$$
\chi=\frac{\sigma(u+v)}{\sigma(v)} e^{-u \zeta(v)}=1+\sum_{n=1}^{\infty} \gamma_{n} \frac{u^{n}}{n !} .
$$

The polynomials $\Gamma_{n}=(x+\gamma)^{n}$ are the coefficients in the expansion

$$
\Theta=\chi \exp x u=1+\sum_{n=1}^{\infty} \Gamma_{n} \frac{u^{n}}{n !},
$$

and may be determined successively by means of the relation

$$
\Gamma_{n}=\frac{\partial \Gamma_{n-1}}{\partial v}-(n-1) \wp(v) \Gamma_{n-2}+x \Gamma_{n-1},
$$

which is a consequence of the fact that $\Theta$ satisfies

$$
\frac{\partial \Theta}{\partial u}-\frac{\partial \Theta}{\partial v}=(x-\wp(v)) \Theta .
$$

* See Tannery-Molk, Fonctions Elliptiques, vol. 3, p. 137. 
Further, since

$$
\frac{\partial \Gamma_{n}}{\partial x}=n \Gamma_{n-1}
$$

we have, by (16), the partial differential equation satisfied by these polynomials, namely,

$$
\wp(v) \frac{\partial^{2} \Gamma_{n}}{\partial x^{2}}-\frac{\partial^{2} \Gamma_{n}}{\partial x \partial v}-x \frac{\partial \Gamma_{n}}{\partial x}+n \Gamma_{n}=0 .
$$

Moreover, it follows as a consequence of the identity

$$
\Theta=\psi \sigma(u)
$$

that the polynomials $\Gamma_{n}$ are expressible linearly in terms of the $A_{n}$. In fact,

$$
\begin{aligned}
& \Gamma_{n}=A_{n}+n ! \sum_{r=1}^{[(n-3) / 2]} \frac{b_{r+1}}{(2 r+3) !} \frac{A_{n-2 r-2}}{(n-2 r-2) !} \\
&+\epsilon(n) \frac{b_{n / 2}}{n+1}, \quad \quad(n=1,2,3, \cdots),
\end{aligned}
$$

where $\epsilon(n)$ is zero or unity according as $n$ is odd or even, $b_{r+1} /(2 r+3)$ ! is the coefficient of the general power in the expansion of the sigma function about the origin, ${ }^{*}$ and $A_{s}=0$ in case $s<0$. If in this result we set $x=0$, we obtain a relation between the two umbras $\alpha$ and $\gamma$.

5. Conclusion. Other systems of Appell polynomials related to our $A_{n}, \Gamma_{n}$ can be obtained from them by applying the processes indicated by Appell in his memoir. $\dagger$ In particular, the systems generated by the functions $d^{k} \phi / d u^{k}, d^{k} \chi / d u^{k}$ may be readily found. Thus, the general Appell polynomial generated by the second of these is given by

$$
\begin{aligned}
\sum_{\rho=0}^{k}(-1)^{\rho}\left(\begin{array}{l}
k \\
\rho
\end{array}\right) x^{\rho}\left[\left(x+\frac{\partial}{\partial v}\right) \Gamma_{n+k-\rho-1}\right. & \\
& \left.-(n+k-\rho-1) \&(v) \Gamma_{n+k-\rho-2}\right],
\end{aligned}
$$

* Halphen, vol. 1, p. 300. Note that $b_{1}=0$. See also Schwarz's Formeln und Lehrsätze, etc., p. 7. Also, Haussner, Über die Zahlencoefficienten in den Weierstrass'schen $\sigma$-Reihen, Göttinger Nachrichten, 1894.

$\dagger$ † Appell, loc. cit., Annales de l'École Normale Supérieure, 1880. 
which, by (16), may be written in the form

$$
\sum_{\rho=0}^{k}(-1)^{\rho}\left(\begin{array}{l}
\rho \\
k
\end{array}\right) x^{\rho} \Gamma_{n+k-\rho},
$$

and hence is expressible in terms of the $\Gamma_{n}$ directly.

These results would be useful in determining the systems of Appell polynomials generated by a general doubly periodic function of the second kind.

The UnIVERSity of Nerraska

\section{ON DOUBLE RIEMANN-STIELTJES INTEGRALS*}

BY J. A. CLARKSON

1. Introduction. A recent study by Clarkson and Adams $\dagger$ of functions $f(x, y)$ of bounded variation naturally leads one to the consideration of double Stieltjes integrals. The present paper is devoted to the discussion of certain questions concerning such integrals.

Stieltjes defined the symbol

$$
\int_{a}^{b} f(x) d \phi(x)
$$

by means of the sum

$$
\begin{gathered}
\sum_{i=1}^{n} f\left(\xi_{i}\right)\left[\phi\left(x_{i}\right)-\phi\left(x_{i-1}\right)\right], \\
\left(a=x_{0}<x_{1}<x_{2}<\cdots<x_{n}=b, x_{i-1} \leqq \xi_{i} \leqq x_{i}\right) .
\end{gathered}
$$

If this sum approaches a finite limit when the norm of the subdivisions approaches zero, (1) is defined as this limit; otherwise (1) is not defined. He showed that for a given $\phi(x)$, a sufficient condition that (1) should exist for every continuous function

* Presented to the Society, December 27, 1933.

$\dagger$ J. A. Clarkson and C. R. Adams, On definitions of bounded variation for functions of two variables, Transactions of this Society, vol. 35 (1933), pp. 824854. 